\title{
RESEARCH ON SHIP AUTONOMOUS STEERING CONTROL FOR SHORT-SEA SHIPPING PROBLEMS
}

\author{
Sergej Jakovlev ${ }^{1}$, Arūnas Andziulis², Andrius Daranda ${ }^{3}$, Miroslav Voznak ${ }^{4}$, Tomas Eglynas ${ }^{5}$ \\ ${ }^{1}$ Marine Science and Technology Centre, Kalipeda University, Lithuania \\ ${ }^{2}$ Dept of Informatics and Statistics, Klaipeda University, Lithuania \\ ${ }^{3}$ Dept of Telecommunications, Technical University of Ostrava, Czech Republic \\ ${ }^{5}$ Dept of Transport Technological Equipment, Vilnius Gediminas Technical University, Lithuania
}

Submitted 14 February 2014; resubmitted 9 February 2015, 13 April 2015; accepted 7 September 2015

\begin{abstract}
Today most ship rotation angle (steering control during movement) increase or decrease is done using an operator on deck or the auxiliary system in the ships engine room. Formal regulations suggest using manual inspection of the ship rotation and the work effectiveness of the engine during manoeuvring in ports and in the open sea regions. The accuracy of this procedure is very low and depends on the personnel of the deck. Therefore, automation and computer control systems are constantly required to assist the human eye. This problem becomes clearly visible when dealing with full ship autonomy in the open sea in the short-sea shipping regions. The trend of maritime technology development will only increase in the area of human interaction decrease with the physical operations and the shipping procedures, which will lead to the future full ship autonomy in the open sea regions around the globe. With the growing automation technologies, predictive control can prove to be a better approach than the traditionally applied visual inspection policy and linear control models. Ship full autonomy is also linked to the ship's machinery regular repair and maintenance that has to be carried out for delivering satisfactory performance and minimizing downtime during transportation operations. In this paper, current stages of development of the intelligent transportation system concept are discussed for the ship autonomy in manoeuvring control and a robust ships' systems integration and communication system concept is presented for several normal and abnormal situations: high-traffic, potentially dangerous situations or port approaching or ship maintenance, with the capability to solve problems with the limited human interface and with a remote control possibility. Then, simplified ship steering motor system for the main pump is analysed for rotation control using control voltage from the converters. Retrieved data from a small experimental control motor is used for the predictive control approach using two different methods: a neural network trained with Basic LevenbergMarquardt Method and a Linear Model.
\end{abstract}

Keywords: transportation; predictive control; human error; communications systems; multi-layer perceptron neural network; intelligent transport system.

\section{Introduction}

The short-sea waterborne transportation makes the use of several systems and technologies, which are not interconnected neither interoperable, including inner ship mechanical and information, communication systems and complex alert situation and terrorist attack prevention systems located both in ports, inland and on-board the ships (Tzannatos, Kokotos 2009). Each single system and piece of technology delivers complex data, other information and functionalities which are vital for the working stability of all waterborne transport operations. The integration of specific decision support functionalities based on Information and Communication Technologies (ICT) in the intelligent ship management systems could deliver new business functionalities and indeed make waterborne transportation more autonomous, efficient and safer (Jakovlev et al. 2013; Goulielmos et al. 1997).

Marine operations have traditionally been managed by professional mariners whose years of experience and training have prepared them for all possible emergency scenarios in the open sea regions of the world, especially in the near land routes and in the short sea shipping. Despite all that, human errors are just as likely to occur when dealing with extreme situations and harsh working environment on-board the ships. The development of new computer control systems and programs, used in remotely controlling vessels in many shipping regions of the world, is now a reality.

Corresponding author: Sergej Jakovlev

E-mail: s.jakovlev.86@gmail.com 
New research in ITC includes Chen et al. (2009). They proposed an autonomous ship design for cleaning the garbage floating on a lake and Do (2011) presented a constructive method to design cooperative controllers that forced a group of under-actuated ships, with limited sensing ranges, to perform a desired formation, and guaranteed no collisions among the ships. Also, Hong et al. (1999) discussed a new autonomous ship collision free trajectory navigation and control system with a new recursive navigation algorithm based on analytic geometry and convex set theory for ship collision free guidance. Indeed, further research of Olenderski et al. (2006) and Toledo et al. (2009) show possibilities to increase the adaptability, availability and autonomy of the waterborne transportation through enhanced autonomy for ship systems and ships by using wireless communication technologies for ship-to-ship and ship-to-shore communications.

Providing and enhancing the storage and interchange of different information, such as technical, administrative, commercial, environmental and navigational information increases the autonomy of ships as well as their adaptability and availability. Therefore, safety and security, efficiency and quality levels of various transport operations increase as well.

On the other hand, developing such system poses significant challenges concerning major ship requirements and scenarios as well as their replicability to a variety of other ships and operations.

- The first challenge is the efficiency of the system, in terms of the task analysis and allocation of tasks for running the system, and thus its cost. Currently, a typical ship operations scenario requires multiple crew and shore personnel to work together, and is thus expensive and difficult to coordinate. Therefore, new approaches are proposed to reduce the time and effort required by automating part or most of the tasks of the behaviour of the ships (Shao et al. 2009; Zivi 2005). Taking inspiration from the field of autonomous robotics, future research will further develop several tools that enable the development of intelligent, autonomous controllers that drive the behaviour of a large variety of tasks (Lee et al. 2010a). Where Lee et al. (2006) proposed an internet-based ship technical information management system to accumulate, manage, share and use various distributed applications and information used for ship design and building and Lee et al. (2010b) discussed a new mobile robotic ship welding system.

- The second challenge is the readiness of response of the automated ship when facing changing situations as a result of a crew members or other personnel or ships' actions. This requires that the controllers are able to act in real-time, while continuing the execution of the assigned tasks. To achieve this goal, modern ship Mechatronics behaviour-based systems make use of an effective approach to robotic and autonomous agent con- trol due to its modularity and robust real-time properties (Roberts et al. 2003).

- The third challenge is the realism of the behaviour of the autonomous ship. Thus, this requirement imposes new constraints on how the ships' underlying behaviours are carried out, in contrast with typical behaviour-based systems in which almost any behaviour that achieves the desired goals is good.

- The fourth and last challenge, but not least, is the understanding of the key elements and the assessment of new technology trends and information services, in legal (liability), safe and economic terms.

All these challenges must take into account various managements, ICT and work regulation variables, including the following processes and procedures, but not limited to them: monitoring of the cargo, traffic management, communication and technologies, weather routing and energy monitoring, safety, monitoring and reporting, port management and predictive maintenance, remote control of operations. Predictive maintenance is a different problem. Marine installations and shipping vessels are highly valuable assets and during the period of their service life they need regular repair and maintenance to be carried out for delivering satisfactory performance and minimizing downtime. Maintenance expenses account for a considerable amount of total operating expenditure of a vessel. By alerting maintenance teams to material fatigue, premature parts wear, water damage, defective machine components and/or poor heat distribution, condition monitoring services provide facilities with the data they need to monitor trouble spots, order replacement parts, and schedule repairs. This advance notice helps facilities take a more proactive approach to maintenance, easing the strain on limited facility budgets and encouraging greater facility safety. One may also notice that this may limit the human interaction in the processes but also increase the human machine systems development and integration. With the advancement in the field of shipping technologies, the vessels are becoming more and more sophisticated in terms of their design and installed equipment on-board. The complexity and the multitude of the equipment found on the vessels need a very effective maintenance plan. The introduction of instrumentation and control techniques, e.g. used for unmanned machinery spaces, has led to their adoption of marine applications. The use of such and more advanced techniques can be extended for the data collection and analysis tasks required for applying the predictive maintenance program for a vessel. With the current market trends and the dynamics of the marine industry state-of-the-art predictive maintenance can provide benefits of reduced downtime, increased asset availability and cost effective maintenance strategy for marine installations.

Regarding the development and marketing of the ICT challenges, various research and industrial projects were launched in the EU (see Table). 
Table. Review of research and industrial projects

\begin{tabular}{|c|c|c|}
\hline $\begin{array}{c}\text { Processes or } \\
\text { operations }\end{array}$ & Project acronyms & Proposed solutions \\
\hline \multirow{3}{*}{$\begin{array}{l}\text { Monitoring } \\
\text { of the cargo }\end{array}$} & MULTITRACK & $\begin{array}{l}\text { Developed and marketed a European service to allow different players to monitor, track } \\
\text { and locate a shipment throughout the whole logistic chain, whatever the means of } \\
\text { transport is used (road, rail and sea), in the most transparent way. }\end{array}$ \\
\hline & OCTOPUS & $\begin{array}{l}\text { Provided some interesting tools for adaptive tracing of communication channels and } \\
\text { tracking of general cargo through all the logistics chain. This is linked with the creation } \\
\text { of a two-layered open and common distributed electronic platform. }\end{array}$ \\
\hline & EWTIS & $\begin{array}{l}\text { The system was designed to provide an information system for the exchange of } \\
\text { electronic data interchange based on messages between community ports and Search } \\
\text { And Rescue (SAR) organisations regarding ships carrying dangerous cargo. }\end{array}$ \\
\hline \multirow{4}{*}{$\begin{array}{l}\text { Traffic } \\
\text { management }\end{array}$} & IPPA & $\begin{array}{l}\text { The IPPA solution provided a visualisation and communications tool for pilots and } \\
\text { masters within congested waters, fairways and river/canal and port approaches and aid } \\
\text { the safe and efficient pilotage of vessels in coastal waters and areas outside Vessel Traffic } \\
\text { System (VTS) coverage, where navigation is constrained. }\end{array}$ \\
\hline & MOVIT & $\begin{array}{l}\text { Studied the realisation of a mobile Vessel Traffic Management Information System } \\
\text { (VTMIS) system compatible with major European VTS systems. }\end{array}$ \\
\hline & TAIE & Defined some tools to assess VTSs and to increase the efficiency of VTS. \\
\hline & POSEIDON & $\begin{array}{l}\text { Tried to achieve a maximum of synergies between the applications and tried to support } \\
\text { and improve the European concept of moving from VTS to VTMIS. }\end{array}$ \\
\hline \multirow[b]{2}{*}{$\begin{array}{l}\text { Communication } \\
\text { technologies }\end{array}$} & ARETOPS & $\begin{array}{l}\text { Studied and then improved the general architecture of the information exchanges by } \\
\text { providing an open technology specific to the maritime sector enabling clearer exchanges } \\
\text { of information. }\end{array}$ \\
\hline & VASME & $\begin{array}{l}\text { Automated some of those information flows. However, this would require a centralised } \\
\text { management of all the needed information. Such a system would provide on-board } \\
\text { systems with a single point of access to all external communication devices and } \\
\text { automate communication management as well as enabling connection to distant } \\
\text { networks. }\end{array}$ \\
\hline $\begin{array}{l}\text { Weather routing } \\
\text { and energy } \\
\text { monitoring }\end{array}$ & SEAROUTES & $\begin{array}{l}\text { Bundled the whole knowledge of ship routing. An advanced decision support system } \\
\text { was established in this project based on full scale ship specific responses as well as } \\
\text { improved medium range weather forecasts including synoptic, high precision and real } \\
\text { time satellite data. }\end{array}$ \\
\hline
\end{tabular}

The intellectual merit criterion of this paper encompasses the potential to advance knowledge in the field of intelligent transport systems and their practical application. The proposed research demonstrates how a simplified version of a neural network programmed control unit can be extended and applied to assist the steering of a vessel with a predictive approach, subject to accuracy bound detection. This technique provides a new application vector for the predictive control algorithms in the field of transport engineering and is intended to raise the efficiency and safety of port and short-sea shipping environments. It is an efficient method for determining accuracy of the prediction using control units for the heavy machinery as well.

\section{Beyond State-of-the-Art and a New Concept}

Because of this complexity, lots of parameters have to be taken into account and the development of the maritime transport and of the rules applied as well as the technological changes create the needs for more and more targeted projects. This is why many research projects have been launched in the past years at European, National and International levels. All those projects could be seen as an endless intent to reach the unreachable. However, these projects also contribute to the general understanding of the feasibility of the intelligent shipping and the e-ship, and in any case for the improvement of the shipping business. The maritime navigation and information systems developed within those projects provided more and more value added services for ships, states, companies, national agencies. E-navigation systems are usually defined in a very broad way for the autonomous ship. However, they can be divided in three main categories:

- the on-board equipment;

- the external information systems and onshore applications;

- the telecommunication and localisation systems, a huge area which can be further divided in two: the telecommunications and earth observation systems on one hand, and the localisation and navigation systems on the other.

Of course, it is a bit diagrammatic since the frontiers between these different kinds of systems are not completely tight and some of the components of one might be closely linked to another, but a line had to be drawn somewhere. For each one of those systems, 
some services have to be provided. For the telecommunication and localisation systems, the key points are seamless tracing and tracking of vessels and hazardous materials, tracing and tracking on long distance, early identification of ships coming into the specific region's waters, traffic surveillance and traffic management. The on-board equipment category is mostly made of technologies that help to better navigate and monitor the different things happening at any time in the fields of communication, weather survey, security, safety and alert. The external information systems are connected to the development and integration of river management, environmental monitoring, freight transport management and port management systems. The impact of improved communication will affect port objectives with relevance to problems solving (cooperation and communication in emergency situations; de-escalation methods; reaction to changing circumstances; Initial and subsequent response measures; combined safety and security in maritime operations; safe and reliable access to the modern maritime information systems provide the crew with huge amount of information). Some data is redundant and the processing and use of such information is made by the crew in every ship. However, the concepts of the autonomous ship include information transfer not only for the above mentioned purposes, but also for control possibility with a high degree of accuracy. But one may notice that such control may only be used for extreme situations. On a daily basis, ships autonomy is based on its possibility to react to the changing circumstances on its own without interference from the remote operators. Therefore, human factor issues in making proper decisions are critical for maintaining transport operations effectiveness. The objective is to increase the adaptability, availability and the autonomy of the waterborne transportation by creating state-of-the-art communication concept of the autonomous ship with a limited human interface in terms of simple human process interactions. The Fig. 1 presents a high level view of the infrastructure and Fig. 2 presents a six layer view of the data flow within the new concept.

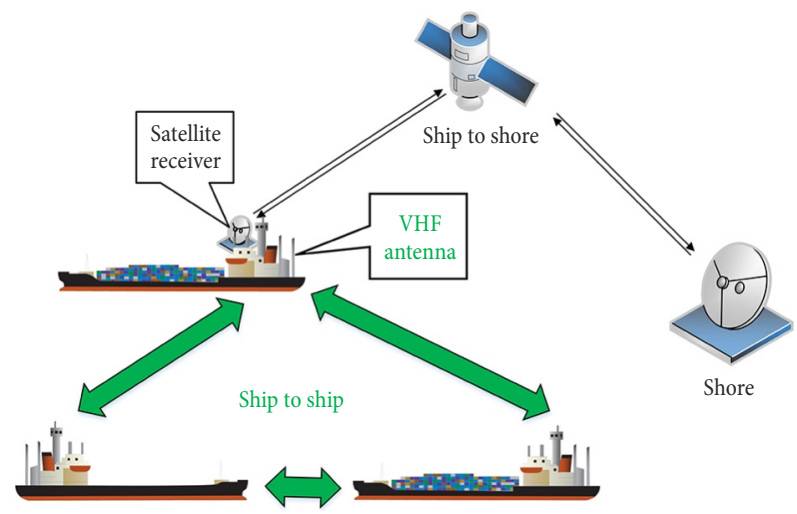

Fig. 1. High level view of the communication network infrastructure
Such integrated intellectual data communication network for the developed short-sea-shipping maritime information system will ensure high adaptability and availability of the information for the operations management and other value added services (safe and reliable access to the internet) through the specific human machine systems. However, there is still room for improvement on this important issue. The most obvious one is the improvement of the exchange means themselves. E-Navigation includes the full integration of the following technologies, standards and systems:

- worldwide coverage of navigation areas by Electronic Navigation Charts (ENC);

- reliable and redundant electronic Global Positioning System (GPS);

- technological structures for transmission of navigational and positional information from ship-to-shore and ship-to-ship (Worldwide Interoperability for Microwave Access (WiMAX), Inmarsat).

Moreover, there are other information systems (e.g., AIS, IBS) that currently are in use by maritime organizations. Bearing in mind its original purpose of ship collision avoidance, the Automatic Identification System (AIS) is an automated tracking system used on ships and by VTS for identifying and locating vessels by electronically exchanging specific identification data with other nearby ships and VTS stations. One possible concrete implementation refers to the innovative and existing navigation automation control system. Very few systems exist on the market, a networked system architecture, where components are encapsulated in functional entities for efficient data distribution around the network.

This architecture can be easily extended by attaching components (sensors, navigation, and communication components) to the network whose data can be accessed from any networked location in the ship. Through an extensive library of standardised function blocks, the system would provide dedicated automation systems according to the vessel requirements. By careful utilization of shared components throughout system hardware and software, this architecture would offer complete consistency in operation and design, e.g., alarm handling is simplified between applications due to common programming. Integration with almost all components required in today's maritime market: from throttle valves, pumps and fans through to tank gauging, PID regulators and similar (Fig. 3). Improvement of marine transportation operations is based on the development and implementation of the 'intelligent ship' concept and addresses the following integration aspects or dimensions: (i) physical integration of infrastructure/ equipment, (ii) information integration through the provision of integrated information services to crew and other stakeholders, and (iii) automated and autonomous tasks within system operation. The major prerequisite for the development of the concept should be the task analysis and human factors integration, in the ship 'system' landscape. 


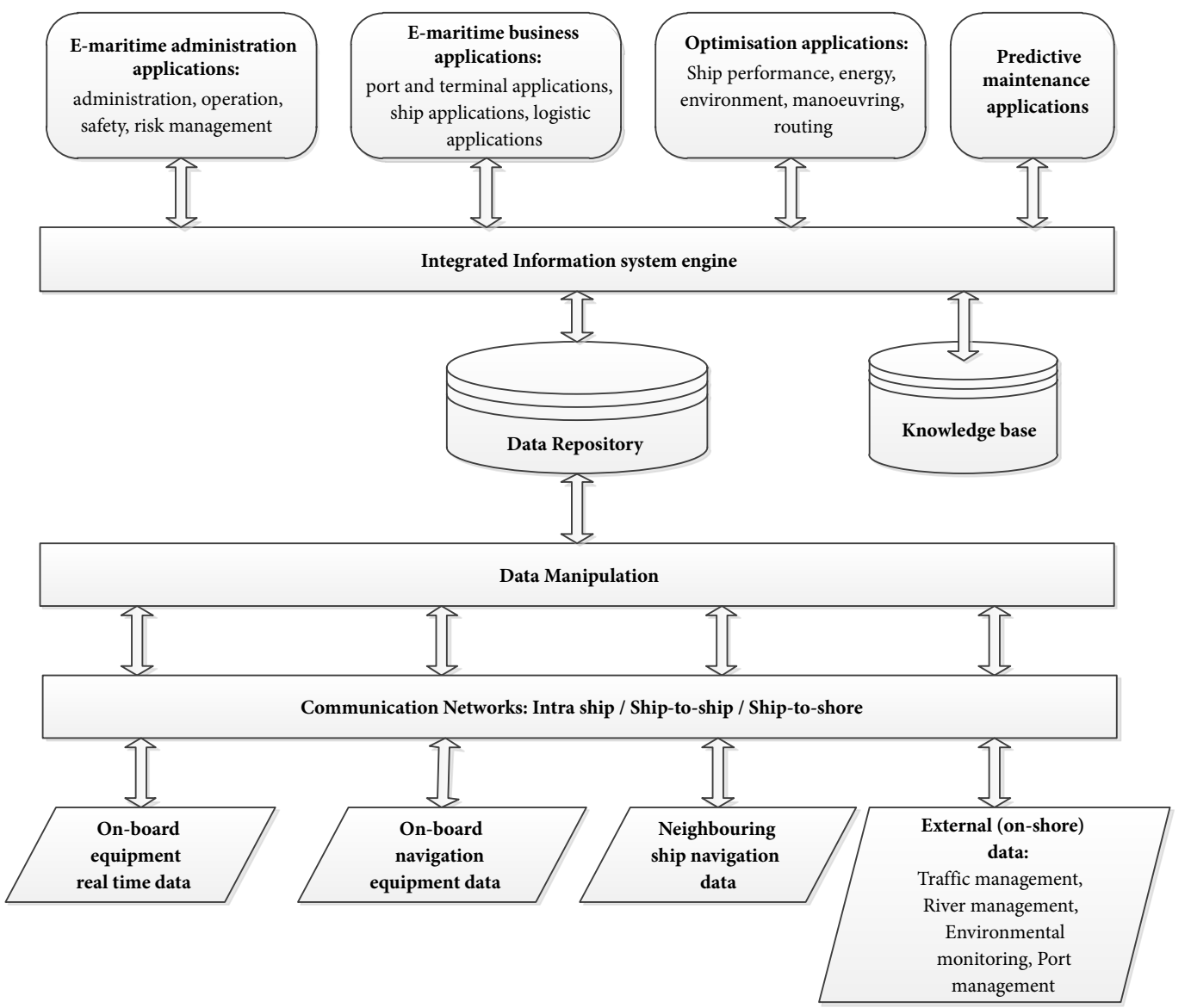

Fig. 2. Integrated system framework



Fig. 3. Integration with networked system architecture 
The next coming step is to define more precisely each one of those already mentioned services. Following research steps concern requirements for integration: the objective is to adapt the operational and business process of the system by integrating these new technologies in order to improve the global system and the quality and autonomy of shipping and ships' operations. Scenarios are drawn from which a system can be integrated and functional:

- scenario 1 - reporting and port clearance;

- scenario 2 - environmental concerns and energy;

- scenario 3 - vessel traffic and navigation;

- scenario 4 - crisis management and abnormal situation management.

The objective is to design information systems for such scenarios, which provide the relevant information in a user-friendly and automated way. The problem of information overflow, redundant and low-quality information is addressed in this context. The next step is to identify the information needs for the scenarios in question, and to create an appropriate ontology for it. The communication between cruise ship passengers, cargo ships' crews and the organisations and the rest of the world ashore (internet) is a great technological and management challenge in the shipping industry. The problem becomes clearly visible when a large amount of data is needed to be transferred from ship-to-ship/ ship-to-shore e.g., (when dealing with the 'autonomous' ship problems (remote monitoring and control of technological processes on-board). Recent developments in wireless communication introduced the possibility to use intellectual data communication ad-hoc network structures. Such innovative approach will help sustain the economic development of communication and business services infrastructure, create new job opportunities and promote not only the advancement of new technology skills, but also the development of new high-tech Small and Medium Enterprises (SMEs), particularly in the advanced ICT transport technologies and servicesoriented activities.

\section{Ship Steering Control under the Autonomy Concept}

\subsection{Description of the Current Situation}

Ships autonomy depends heavily on the ships capability to react to the changing circumstances in a timely manner and with a low error possibility. Such heavy machinery control can be achieved using various innovative methods and technologies. The best solution is to use predictive control method with a programmed neural network algorithm. These methods proved to be very effective in many cases and in different control areas, ranging from ship inner valves control to partial steering control in average day situations. Most ships have steering control mechanisms with a simple wheel and a control subsystem with a switch desktop in the engine rooms, so there is no agility in control (Figs 4 and 5). In terms of this paper, we also suggest that this future trend will also affect all current vessels in operation.
Otherwise, the autonomy concept will be useless in many situations in the short-sea regions of the world. The majority of ships that are used by companies have quite old inner installations and their constant inspection is required. The situation described in Fig. 4 also describes the complications that arise with the monitoring and maintenance of old installations.

\subsection{Research on the Predictive Control}

In this sub-section a simplified ship steering motor system for the main pump is analysed for rotation predictive control using control voltage from the converters (Fig. 5).

Retrieved data from a small experimental DC motor is used for the predictive control approach using two different methods: a neural network trained with Basic Levenberg-Marquardt Method and a Linear Model. A MultiLayer Perceptron (MLP) network approach is used for prediction of the indicator voltage output which affects the monitoring of the rotation and steering effectiveness.

\subsubsection{Control Problem}

Simple computer based prediction of control motor speed indicator voltage output can be used to minimize the time for the steering process to react with the pump system, physical ship steering mechanism and limit unwanted mechanical corrections. Much like in the research of Ferreau et al. (2007) and Wang et al. (2006), such predictive control tool can also prove to be a valuable addition to the existing systems as well. Received parameters can be used not only for control, but also for maintenance purposes analysed previously in this work for the old ship systems. If this prediction tool can predict voltage fluctuation that has a direct impact on the motor rotation speed and therefore the pump work, then the same data can be used to signal that a malfunction may occur in this system. Predictive control of the machinery also links to predictive maintenance and neural network models (Vasičkaninová et al. 2011; Vahidinasab et al. 2008). The work of Savran et al. (2006) was the most noticeable.

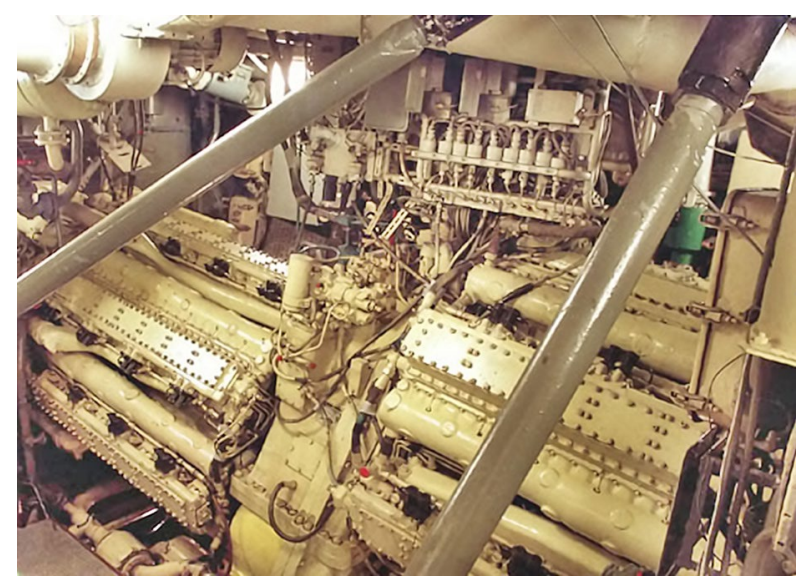

Fig. 4. Example of an old ships engine room 


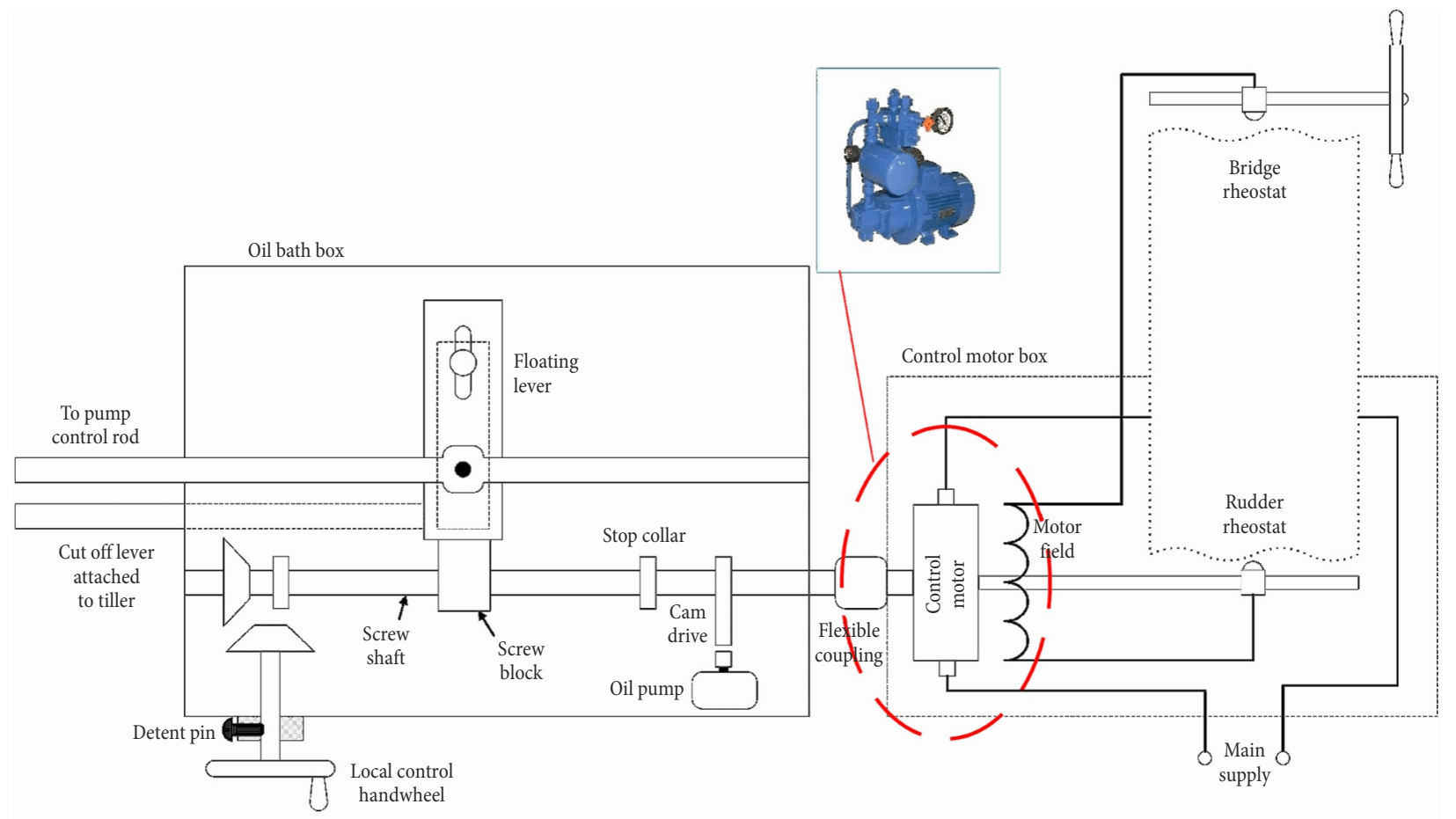

Fig. 5. Schematic example of the ships steering system with the indicated experimental control motor for pump

In their work authors described the development of a neural network based on adaptive flight control system for a high performance aircraft. Their proposed system was able to compensate the system uncertainties, adapt to the changes in flight conditions, and accommodate the system failures in real time. To speed up the convergence rate and to enhance the accuracy for achieving the online learning, they adapted the Levenberg-Marquardt optimization method with a trust region approach to train the neural network. Similar techniques can prove to be efficient to control the disk rotation angle in real time. Overall, ships installations are highly valuable assets and during the period of their service life they need regular repair and maintenance to be carried out for delivering satisfactory performance and minimizing downtime. Currently, visual maintenance expenses with human interaction account for a considerable amount of total operating expenditure. As the equipment begins to fail, it may display signs that can be used to identify the onset of equipment degradation and potential failures in control. As an example, metal corrosion can cause the rotating parts of the system to fail and to work with a high margin of error. This can influence the effectiveness of the autonomous ship in terms of navigation, route planning and immediate reaction to engine failures in the short-sea shipping areas.

\subsubsection{Analysis of the Linear Model}

The system uses several technologies. In this paper, we present the multi-layer perceptron neural network approach to analyse the working condition of small control motor. A neural network was used for the prediction of the voltage fluctuation. This fluctuation can have a serious effect on the working stability and measurement results, and the autonomy of the ship in terms of route and e-navigation stability. These fluctuations occur when steering control motor is controlled and its operation is constantly corrected. Like in older ships, due to the poor condition of the installations, some grid noise and other deviations in the voltage output measurements occur. 1 Volt control voltage was presented to the converter for the small control motor. When control and indicator values are fluctuating around 0 Volts is the period of time when the engines are not manually launched from the control desktop. The same control process is performed constantly when steering is necessary.

Next, we tried to apply a Linear Model. If it does a good job, one should not bother wasting time on neural network based model structures like MLP. We identified a linear Output-Error (OE) polynomial model using time-domain data with discrete-time IDPOLY model (polynomial model with identifiable parameters) estimation on the output. In our case an IDPOLY model represents a system as a continuous discrete-time polynomial model with identifiable (estimable) coefficients A, B, C and $\mathrm{D}(1)$. In our case, the simple discrete-time polynomial model estimation can be presented as (1) with input vector $u$, output vector $y$, and disturbance (error) $e$ takes the following form in discrete time:

$$
A(q) y(t)=\sum_{i=1}^{n u} \frac{B_{i}(q)}{F_{i}(q)} u_{i}(t)+\frac{C(q)}{D(q)} \cdot e(t) .
$$

And the OE model is estimated, and presented by a general model (2) with time delays:

$$
y(t)=\sum_{i=1}^{n u} \frac{B_{i}(q)}{F_{i}(q)} u_{i}\left(t-n k_{i}\right)+e(t) .
$$


Where the orders of the OE model are ( $n b: 3, n f: 4)$ :

$$
\begin{aligned}
& B(q)=b_{1}+b_{2} q^{-1}+\ldots+b_{n b} q^{-n b+1} ; \\
& F(q)=1+f_{1} q^{-1}+\ldots+f_{n f} q^{-n f} .
\end{aligned}
$$

The orders $n b$ and $n f$ parameterize the estimated polynomial alongside with other estimators in the MATLAB OE model and IDPOLY model evaluation syntax and $n k$ is the delay. Model transfer function is defined as (5):

$$
T F\left(q^{-1}\right)=z^{-k} \frac{B\left(q^{-1}\right)}{F\left(q^{-1}\right)} .
$$

In discrete time, $z^{-k}$ represents a time delay of $n k$. In our case, time delays are constant. The general polynomial equations presented above are written in terms of the time-shift operator $q^{-1}$. The prediction is the smooth curve while the noisier one represents the measurements (Fig. 6).

Next we validated an estimated Linear Model, by displaying the auto correlation function of the residuals and the cross correlation function between input and residuals. From Fig. 6 we can assume that the Linear Model has many problems regarding its accuracy, especially for large magnitudes. Therefore, a conclusion is made that this is due to the underlying system being nonlinear.

\subsubsection{Review of MLP Network}

In this paper we use an MLP network that has only one hidden layer and only hyperbolic tangent and linear activation functions $(f, F)$ in the form of (6):

$$
\hat{y}_{i}(w, W)=F_{i}\left(\sum_{j=0}^{q} W_{i j} f_{j}\left(\sum_{l=1}^{m} w_{j l} z_{l}+w_{j 0}\right)+W_{i 0}\right) .
$$

The weights (specified by the vector $\theta$ and alternatively by the matrices $w$ and $W$ ) are the adjustable parameters of the network, and they are determined through network training. The training data is a set of inputs $u(t)$, and corresponding desired outputs $y(t)$. The training set specified by (7):

$$
Z^{N}=\{[u(t), y(t)] \mid t=1, \ldots, N\} .
$$

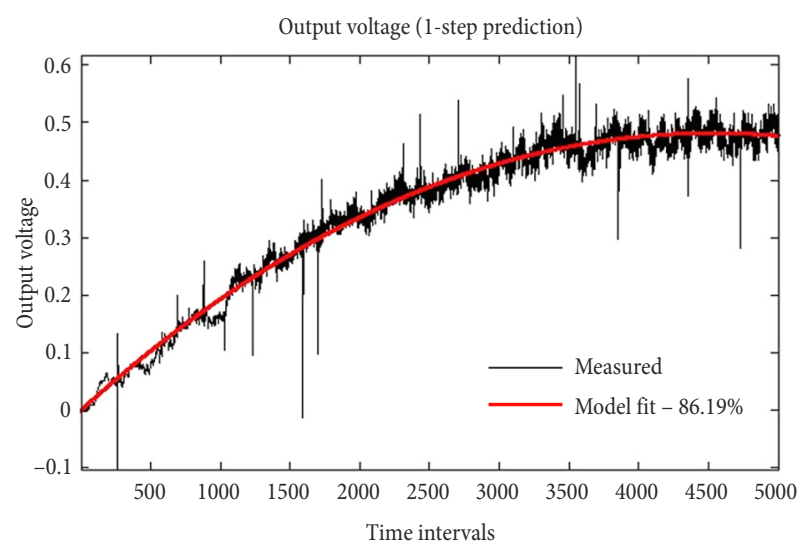

Fig. 6. Linear model fit
The objective of training is then to determine a mapping from the set of training data to the set of possible weights (8):

$$
Z^{N} \rightarrow \widehat{\theta} .
$$

The network will produce predictions $\hat{y}(t)$, which in some sense are close to the true outputs $y(t)$. The prediction error approach, which is the strategy applied in this paper, is based on the introduction of a measure of closeness in terms of a mean square error criterion (9):

$$
\begin{aligned}
& V_{N}\left(\theta, Z^{N}\right)=\frac{1}{2 N} \times \\
& \sum_{t=1}^{N}[y(t)-\hat{y}(t \mid \theta)]^{T}[y(t)-\hat{y}(t \mid \theta)] .
\end{aligned}
$$

The weights (10) are:

$$
\widehat{\theta}=\arg \min V_{N}\left(\theta, Z^{N}\right) \text {. }
$$



$$
\theta^{(i+1)}=\theta^{(i)}+\mu^{(i)} f^{(i)} \text {. }
$$

where: $\theta^{(i)}$ specifies the current iterate (number ${ }^{\prime} i$ ); $f^{(i)}$ is the search direction; $\mu^{(i)}$ the step size.

The network was trained with Basic LevenbergMarquardt Method as it is a very popular curve-fitting algorithm used in many software applications for solving generic curve-fitting problems (Kermani et al. 2005; Mukherjee, Routroy 2012). It was used for minimization of mean-square error criteria, due to its rapid convergence properties and robustness (Vahidinasab et al. 2008). Some improvements were made to the method $(12,13)$. The size of the elements of the diagonal matrix added to the Gauss-Newton Hessian was adjusted according to the size of the ratio between actual decrease and predicted decrease.

$$
\begin{aligned}
& r^{(i)}=\frac{V_{N}\left(\theta^{(i)}, Z^{N}\right)-V_{N}\left(\theta^{(i)}+f^{(i)}, Z^{N}\right)}{V_{N}\left(\theta^{(i)}, Z^{N}\right)-L^{(i)}\left(\theta^{(i)}+f^{(i)}\right)} ; \\
& L\left(\theta^{(i)}+f\right)=V_{N}\left(\theta^{(i)}, Z^{N}\right)+ \\
& f^{T} G\left(\theta^{(i)}\right)+\frac{1}{2} f^{T} R\left(\theta^{(i)}\right) f,
\end{aligned}
$$

where: $G$ denotes the gradient of the criterion with respect to the weights; $R$ is the so-called Gauss-Newton approximation to the Hessian.

The following algorithm was used:

Step 1: Select an initial parameter vector $\theta^{(0)}$ and an initial value $\lambda^{(0)}$;

Step 2: Determine the search direction from $\left[R\left(\theta^{(i)}+\lambda^{(i)} \cdot I\right)\right] f^{(i)}=-G\left(\theta^{(i)}\right), I$ being a unit matrix;

Step 3: $r^{(i)}>0.75 \Rightarrow \lambda^{(i)}=\lambda^{(i)} / 2$, if predicted decrease is close to actual decrease let the search direction approach the Gauss-Newton search direction while increasing step size;

Step 4: $r^{(i)}<0.25 \Rightarrow \lambda^{(i)}=2 \lambda^{(i)}$, if predicted decrease is far from the actual decrease let the search direction approach the gradient direction while decreasing step size; 
Step 5: If $V_{N}\left(\theta^{(i)}+f^{(i)}, Z^{N}\right)<V_{N}\left(\theta^{(i)}, Z^{N}\right)$ then accept $\theta^{(i+1)}=\theta^{(i)}+f^{(i)}$ as a new iterate and let $\lambda^{(i+1)}=\lambda^{(i)}$ and $i=i+1$;

Step 6: If the stopping criterion is not satisfied go to Step 2).

Next step is to select structure of the model. It is necessary to choose a set of regressors and to determine network architecture. The idea is to select the regressors based on the linear system and then determine the network architecture with the given regressors as inputs. In this work the following regressors (14) and the predictor vectors (15) are used:

$$
\begin{aligned}
& \varphi(t)=\left[\begin{array}{l}
\hat{y}(t-1 \mid \theta) \ldots \hat{y}(t-n a \mid \theta) u(t-n k) \ldots \\
u(t-n b-n k+1)
\end{array}\right]^{T} ; \\
& y(t \mid \theta)=a(\varphi(t), \theta),
\end{aligned}
$$

where: $t$ specifies sampling instant number $t ; \varphi(t)$ is a vector containing the regressors; $\theta$ is a vector that contains the weights; $g$ is the function realized by the neural network; $n a, n b, n k$ are used in order for the function to be able to determine the structure of the regression vector used in multi-output and multi-input systems.

\subsubsection{Neural Network Model for Predictive Control}

Firstly, we selected fully connected network architecture with 10 hidden hyperbolic tangent units (Fig. 7). The network was trained with 1000 iterations and with small weight decay. The function initialized the weights. The influence of the unknown initial conditions was also reduced. Next, validation of the trained network was performed. The following results were achieved (Figs 8 and 9). The achieved results are definitely better than the Linear Model.

By comparing the plots for training and test set, we can see that the network is over-fitting the data at a certain degree. Therefore, the selected model structure contains too many weights and the in future applications it should be decreased. In order to remove the unwanted weights and to determine the optimal network architecture, we used a network pruning technique, see Fig. 10 for more detailed results. Network pruning methods are used for optimisation of both the size and the generalization capabilities of the neural network. Fig. 10 displays training error, test error, and Final Prediction Error (FPE) estimate of the generalization error of each of the intermediate networks.

This figure shows that the minimum of the test error occurs when there are only 37 weights left on the network. Ideally, one would expect the training error to increase monotonically as the weights are pruned, while FPE estimate and test error should decrease until a certain minimum, and the start increasing as well. However, the final results depend on the initial local minimum and the initial weights.

Therefore, based on the above research, it is clearly visible that by utilizing some new techniques and tech-

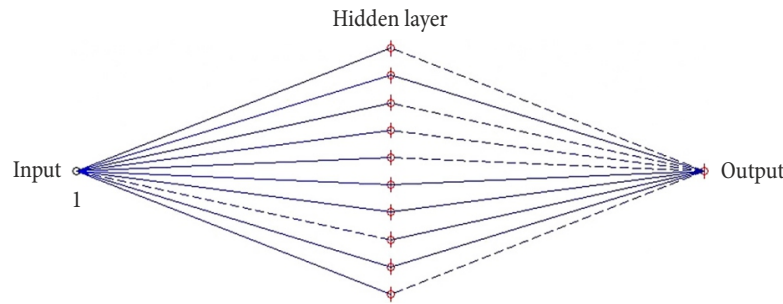

Fig. 7. Final trained neural network (example provided for presentation) with the best learning results


Fig. 8. Neural network model fit

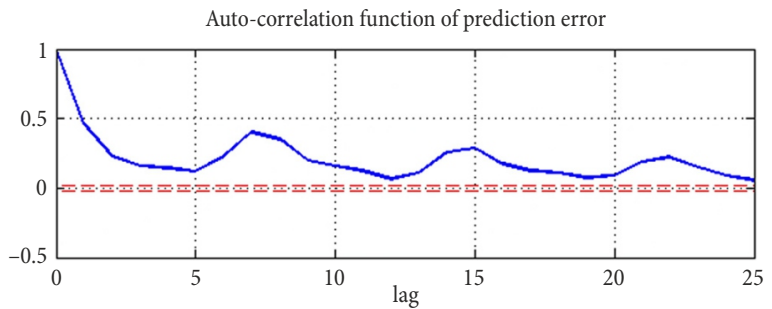

$\times 10^{-3} \quad$ Cross-correlation coef. of $\mathrm{ul}$ and prediction error

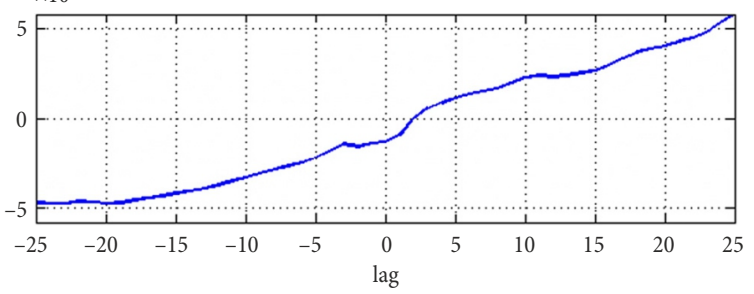

Fig. 9. Model correlation plot

nologies for steering control, we can sufficiently improve the possibility of e-navigation in terms of operations autonomy and prediction. Application of neural network structures for this matter is not an innovation, but the application in total, presented in the introduction section, states clearly that autonomy can only be achieved by utilizing all state-of-the-art concepts in a single system. Innovation can then be achieved not in the instruments themselves but with their integration and specific application. A closer look at the results demonstrates that it is possible to operate under nor- 


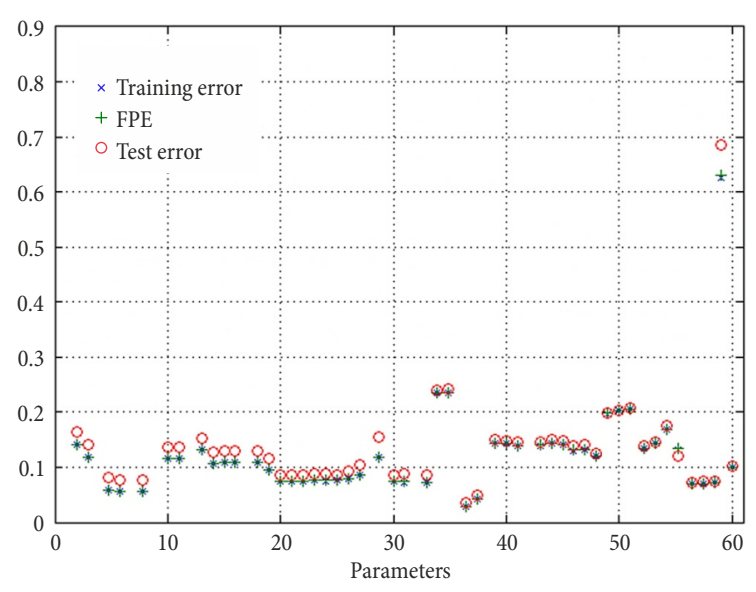

Fig. 10. Network pruning results

mal time boundaries in relation to e-navigation. Simple computations and their results are achieved with a high accuracy level in due time. On the other hand, much computation speed can be achieved for the prediction if the computational resources are high. Then, deviation in the model correlation plot would decrease and Wrights analysis would suggest using less weight. That means, less time consuming.

\section{Conclusions}

In this paper, we have analysed a complex problem of predictive control of the ships steering motor system for the main pump for ships rotation control using control voltage from the converters. Retrieved data from a small experimental control motor was used for the predictive control approach using two different methods: a neural network trained with Basic Levenberg-Marquardt Method and a Linear Model. A MLP network approach was used for prediction of the indicator voltage output which affects the monitoring of the rotation and steering effectiveness. Data was used to predict 1-step ahead the voltage output, thus disk rotation speed. Two models were proposed and compared. Linear Model proved to be $>86 \%$ effective with the predictions and the MLP neural network proved to be more precise in predictions. In literature many authors suggest that using complex neural networks for predictive control may cause the delay of time for the needed computations to occur. On the other hand, the newly trained neural network will perform much quicker by using less hidden neurons after some initial learning. During the experiment, different network structures were examined with a different number of hidden neurons. Results suggest that a network with the number of neurons $>6$ can perform much better than the Linear Model. Computation of this network takes only a few seconds on the initial training.

Regarding implication of the research, in the late 2014, a new research vessel was launched by Klaipeda University which incorporates both the hardware and software parts for steering predictive control. Exploiting the potential of internet/intranet as well as Information and Communication Technology is likely to be the main route for achieving the goal of the Improvement of Marine Transportation Operations, thus achieving the goal of intelligent and autonomous ships and hence of seamless intermediate and short-sea shipping in both normal and abnormal operating conditions. In the future perspective, integrated technologies will increase the availability of knowledge, data and information in a dynamic environment for ships that can become more autonomous and intelligent, and therefore will contribute to the increase of the overall quality of waterborne transportation and to its role in intermodality and shortsea shipping and as a more sustainable transport system.

In general, it should be taken into account that inaccurate and erroneous data are still a problem with positioning and tracking systems, whilst ICT interoperability is also a challenge.

\section{Acknowledgements}

This work has been supported by the European Social Fund within the project 'Development and application of innovative research methods and solutions for traffic structures, vehicles and their flows', project code VP13.1-ŠMM-08-K-01-020 (Lithuania) and is partially supported by Grant of SGS No SP2015/42, Technical University of Ostrava (Czech Republic).

\section{References}

Chen, S.; Wang, D.; Liu, T.; Ren, W.; Zhong, Y. 2009. An autonomous ship for cleaning the garbage floating on a lake, in Second International Conference on Intelligent Computation Technology and Automation 2009 (ICICTA'09), 10-11 October 2009, Changsha, Hunan, China, 3: 471-474. https://doi.org/10.1109/ICICTA.2009.579

Do, K. D. 2011. Practical formation control of multiple underactuated ships with limited sensing ranges, Robotics and Autonomous Systems 59(6): 457-471.

https://doi.org/10.1016/j.robot.2011.03.003

Ferreau, H. J.; Ortner, P.; Langthaler, P.; Del Re, L.; Diehl, M. 2007. Predictive control of a real-world Diesel engine using an extended online active set strategy, Annual Reviews in Control 31(2): 293-301.

https://doi.org/10.1016/j.arcontrol.2007.09.001

Goulielmos, A.; Tzannatos, E. 1997. Management information system for the promotion of safety in shipping, Disaster Prevention and Management: an International Journal 6(4): 252-262. https://doi.org/10.1108/09653569710179093

Hong, X.; Harris, C. J.; Wilson, P. A. 1999. Autonomous ship collision free trajectory navigation and control algorithms, in 1999 7th IEEE International Conference on Emerging Technologies and Factory Automation: Proceedings. ETFA'99, 18-21 October 1999, Barcelona, Spain, 2: 923-929. https://doi.org/10.1109/ETFA.1999.813090

Jakovlev, S.; Voznak, M.; Andziulis, A.; Kurmis, M. 2013. Communication technologies for the improvement of marine transportation operations, IFAC Proceedings Volumes 46(15): 469-474.

https://doi.org/10.3182/20130811-5-US-2037.00020

Kermani, B. G.; Schiffman, S. S.; Troy Nagle, H. 2005. Performance of the Levenberg-Marquardt neural network training method in electronic nose applications, Sensors and Actuators B: Chemical 110(1): 13-22. https://doi.org/10.1016/j.snb.2005.01.008 
Lee, D.; Ku, N.; Kim, T.-W.; Lee, K.-Y.; Kim, J.; Kim, S. 2010a. Self-traveling robotic system for autonomous abrasive blast cleaning in double-hulled structures of ships, Automation in Construction 19(8): 1076-1086.

https://doi.org/10.1016/j.autcon.2010.07.011

Lee, D.; Lee, S.; Ku, N.; Lim, C.; Lee, K.-Y.; Kim, T.-W.; Kim, J.; Kim, S. H. 2010b. Development of a mobile robotic system for working in the double-hulled structure of a ship, Robotics and Computer-Integrated Manufacturing 26(1): 13-23. https://doi.org/10.1016/j.rcim.2009.01.003

Lee, S.-S.; Lee, J.-K.; Park, B.-J.; Lee, D.-K.; Kim, S.-Y.; Lee, K.H. 2006. Development of internet-based ship technical information management system, Ocean Engineering 33(13): 1814-1828. https://doi.org/10.1016/j.oceaneng.2005.10.019

Mukherjee, I.; Routroy, S. 2012. Comparing the performance of neural networks developed by using Levenberg-Marquardt and Quasi-Newton with the gradient descent algorithm for modelling a multiple response grinding process, Expert Systems with Applications 39(3): 2397-2407. https://doi.org/10.1016/j.eswa.2011.08.087

Olenderski, A.; Nicolescu, M.; Louis, S. J. 2006. A behaviorbased architecture for realistic autonomous ship control, 2006 IEEE Symposium on Computational Intelligence and Games (CIG’06), 22-24 May 2006, Reno/Lake Tahoe, Nevada US, $148-155$. https://doi.org/10.1109/CIG.2006.311694

Roberts, G. N.; Sutton, R.; Zirilli, A.; Tiano, A. 2003. Intelligent ship autopilots: a historical perspective, Mechatronics 13(10): 1091-1103. https://doi.org/10.1016/S0957-4158(03)00044-8

Savran, A.; Tasaltin, R.; Becerikli, Y. 2006. Intelligent adaptive nonlinear flight control for a high performance aircraft with neural networks, ISA Transactions 45(2): 225-247. https://doi.org/10.1016/S0019-0578(07)60192-X

Shao, X.-Y.; Chu, X.-Z.; Qiu, H.-B.; Gao, L.; Yan, J. 2009. An expert system using rough sets theory for aided conceptual design of ship's engine room automation, Expert Systems with Applications 36(2): 3223-3233. https://doi.org/10.1016/j.eswa.2008.01.011

Toledo, N.; Higuero, M.; Jacob, E.; Aguado, M. 2009. A novel architecture for secure, always-best connected ship-shore communications, in 2009 9th International Conference on Intelligent Transport Systems Telecommunications (ITST), 20-22 October 2009, Lille, France, 192-197. https://doi.org/10.1109/ITST.2009.5399359

Tzannatos, E.; Kokotos, D. 2009. Analysis of accidents in Greek shipping during the pre- and post-ISM period, Marine Policy 33(4): 679-684. https://doi.org/10.1016/j.marpol.2009.01.006

Vahidinasab, V.; Jadid, S.; Kazemi, A. 2008. Day-ahead price forecasting in restructured power systems using artificial neural networks, Electric Power Systems Research 78(8): 1332-1342. https://doi.org/10.1016/j.epsr.2007.12.001

Vasičkaninová, A.; Bakošová, M.; Mészáros, A.; Klemeš. J. J. 2011. Neural network predictive control of a heat exchanger, Applied Thermal Engineering 31(13): 2094-2100. https://doi.org/10.1016/j.applthermaleng.2011.01.026

Wang, S. W.; Yu, D. L.; Gomm, J. B.; Page, G. F.; Douglas, S. S. 2006. Adaptive neural network model based predictive control for air-fuel ratio of SI engines, Engineering Applications of Artificial Intelligence 19(2): 189-200. https://doi.org/10.1016/j.engappai.2005.08.005

Zivi, E. 2005. Design of robust shipboard power automation systems, Annual Reviews in Control 29(2): 261-272. https://doi.org/10.1016/j.arcontrol.2005.08.004 